\title{
Congenital Teratoma of Nasopharynx
}

\author{
S. R. Desai, Prof \& Head, V. D. Dombale, Lecturer, N. N. Angarkar, Associate Professor \\ Department of Pathology, Kishna Institute of Medical Sciences, Karad - 415110
}

T eratomas are congenital tumours which occur rarely in head and neck. Germ cell tumours arising primarily in head and neck account for approximately $5 \%$ of all gonadal and extragonadal germ cell tumours in children. The most common location in head and neck is cervical region followed by nasopharynx. They present almost exclusively in neonates form pedunculated or sessile masses causing obstruction of the nasopharynx. Clinical problems include difficulty in breathing, sucking or swallowing.

We report a case of nasopharyngeal teratoma for its huge size, rarity of its incidence and occurrence at an unusual site.

\section{Case Report}

Sminurue age up. She came to hospital with obstructed labour and an emergency casesarean section was done to deliver a male still born baby with a large mass coming out through mouth measuring $15 \times 11 \times 8 \mathrm{cms}$. An autopsy was performed. The mass was arising from sphenoid bone externally and on the cut surface the mass was partial solid and cystic. Microscopy revealed skin with adnexa, fibrous tissue muscle, adipose tissue, cartilage bone and glands representing all the three germ cell layers.

\section{Discussion}

The origin of the term from Greek 'Teraton' or monster reflects a capacity for uncoordinated growth both tumoral and malformative (1) As defined by Willis Teratomas are true neoplasms composed of multiple tissues foreign to the part in which they arise. Based on etymologic derivation the term teratoma denotes disorderly or monstrous growth but does not stipulate which or how many germ layer derivatives must be represented (2)

Teratomas have been classified into 4 types based on the number of germ cell layers represented and the differentiation of tissues. The dermoids are composed of only epidermal and mesodermal elements (2). Teratoids are poorly differentiated tumours containing 3 germ cell layers. The true teratomas are well differentiated tumours containing all 3 germ layers. The epignathi are gross maldevelopment of organs and limbs and are rarely compatible with life.

Microscopic examination shows a variety of tissues from the three germ cell layers with a wide range of cellular differentiation and variable degree of maturation (3). Our case is an example of benign mature true teratoma showing all 3 germ layers.

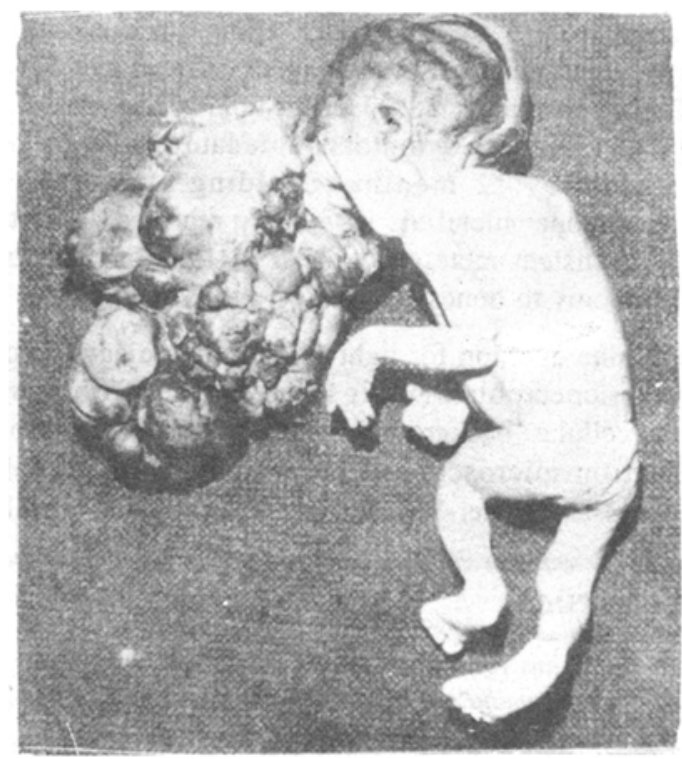

Fig. I Showing a large mass coming out through the mouth cavity

The two most pervalent theories of histogenesis are (1) origin from primordial germ cells in their course of migration during embryogenesis (2) origin from totipotential embryonic cells that have escaped regulatory influences $(2)$.

Where as teratoms elsewhere in the body usually show a predilection for females those in the head and neck reveal an equal sex distribution (3). Our case was teratoma in a male child. 
Grossly teratomas may be solid or cystic. They may be benign or malignant Histologically they may be mature or immature. In contrast to adult teratomas childhood teratoma are invariably benign despite the presence of immature elements.
The differential diagnosis of nasopharyngeal tumours in infants includes craniopharyngioma, chordomas, encephalocoeles, hamartomas, rhabdomyomas, gliomas (3)

Treatment consists of surgical excision.

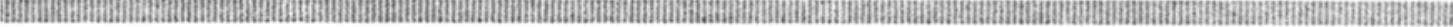

\section{References}

1. Kohli G. S. Yadav S P S et al (1989): Teratoma of the Nasopharynx in the New born Indlan Journal of Otolaryngology $41: 31$.

2. Lack Ernest E. (1985) : Exteragonadal germ cell tumours of Head P Neck region. Human Pathology Vol. 16 No I.

3. 'Yousry EL - SAYED (1992) : Teratoma of the Head \& Neck-The Journal of laryngology \& otologv Sept. Vot 106 836-838

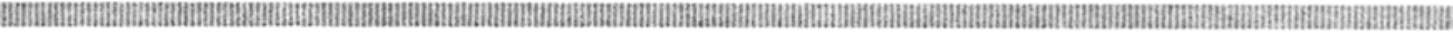

\section{CORRECTIVE RHINOPLASTY}

(with Colour Illustration)

By V. P. Sood

Past-President, Association of Otolaryngologists of India

Rs. 695 , Size : 23 x 29 cms; Pages : 228

\section{ABOUT THE BOOK}

This Book aims at providing guidelines, in a systematic manner on surgical anatomy, applied physiology preoperative assessment and operative techniques of rhinoplasty to the learning surgeon. The main techniques with particular emphasis on the basic steps. The techniques have been illustrated with the help of schematic drawings and clinical photographs.

\section{CONTENTS}

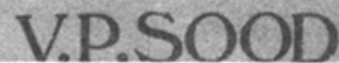

History of Rh inoplasty $\bullet$ Surgical Anatomy and Physiology of the Nose - Pre-Operative Assessment, Aesthetics, Psychological Evaluation and Instrumentation $\bullet$ Surgery of the Bony and Upper Cartilaginous Vaults of Nose $\bullet$ Surgery of the Lower Cartilaginous Vault $\bullet$ Surgery of the Saddle and Crooked $\bullet$ Nose External Rhinoplasty $\bullet$ Septoplasty Reconstructive Rhinoplasty $\bullet$ Mentoplasty $\bullet$ Injuries of the Nose $\bullet$ Postoperative Care, Complications \& Secondary Rhinoplasty $\bullet$ Appendix A : Proforma for recording operative steps • Appendix $B$ : Post-operative instructions to patient
Pages : 228
Size : $2329 \mathrm{~cm}$
ISBN : 81-239-0316-?
Rs. 695

\section{CBS PUBLISHERS \& DISTRIBUTORS}

4596/1A, 11 Darya Ganj, New Delhi 11002 (India)

Tel. 3271632, 3276712 